\title{
Implementation and Usage Evaluation for Self Health Management with App-based Framework
}

\author{
Chen-Shie $\mathrm{HO}^{\mathrm{a}}$ and Yu-Mei CHANG ${ }^{\mathrm{b}}$ \\ 58, Sihchuan Rd. Sec.2, Pan-Chiao District, New Taipei City 22061, Taiwan \\ ${ }^{\mathrm{a}} \mathrm{FL} 007 @$ mail.oit.edu.tw, ${ }^{\mathrm{b}} \mathrm{FK} 001 @$ mail.oit.edu.tw
}

\begin{abstract}
Keywords: Self-care, Healthcare, App
Abstract. This study implements an Android-based self-healthcare App program that integrates the outpatient recommendation system with hospital registration system that allows users to find the information on possible illnesses and corresponding department of treatment for registering. In addition, the value-added services for hospital also are implemented to provide users the flexibility and convenience with self-maintenance of personal health information. The statistical results are evaluated to reflect the usage status by users in this study.
\end{abstract}

\section{Introduction}

Mobile healthcare is in the early stages of explosive growth, and there are endless business opportunities to be developed. How to promote health management through mobile devices will be the future trend [1]-[2]. As the medical technology advances, personal health has been promoted from the disease treatment to the stage of preventive care. At the same time, with the advent of the global age of aging, personal health management is becoming important on care about extending the healthy age than the average life expectancy. Health management services should not only be a health examination, it should also include follow-up health care, disease risk assessment, health care consulting and other related services. It also should extend for population of sub-health, through exercise prescription and diet control to intervene their improvement in life style, to achieve the purpose of health promotion. Many enterprises and organizations have used the network technology to provide fruitful convenient services, which makes modern people's daily life becomes more convenient. At present, most of the medical centers, regional hospitals and district hospitals in Taiwan have set up the hospital website to facilitate people to access many medical information through the website [3]-[4]. Apart from the hospital profiles, medical records and other medical information, many hospitals have begun to provide some more convenient services, such as network registration for people. It can be seen the trend of the population in use of the hospital network registration is upwards. Literature shows that many newly diagnosed people have the experience of misregistration [5]. The reason why people make these mistakes is lacking of medical professional knowledge, and the disease-related information to determine the disease always only exist in professional medical books. The express of knowledge varies according to the author's point of view and for the specific purposes or topics. The result of misregistration includes from waste of patient waiting and traffic time to holding up the medical treatment time and causing both the patient and hospital waste of time and hospital medical costs. Therefore, this study creates a multi-function App that combines a registration system with other practical health services to provide busy users with a real-time action self-care system to achieve both self-health management and time management goals [6]-[10].

\section{System description}

The proposed system structure of App program is shown in Fig. 1, which contains a total of 5 main functions, respectively. These functions are described as follows: 


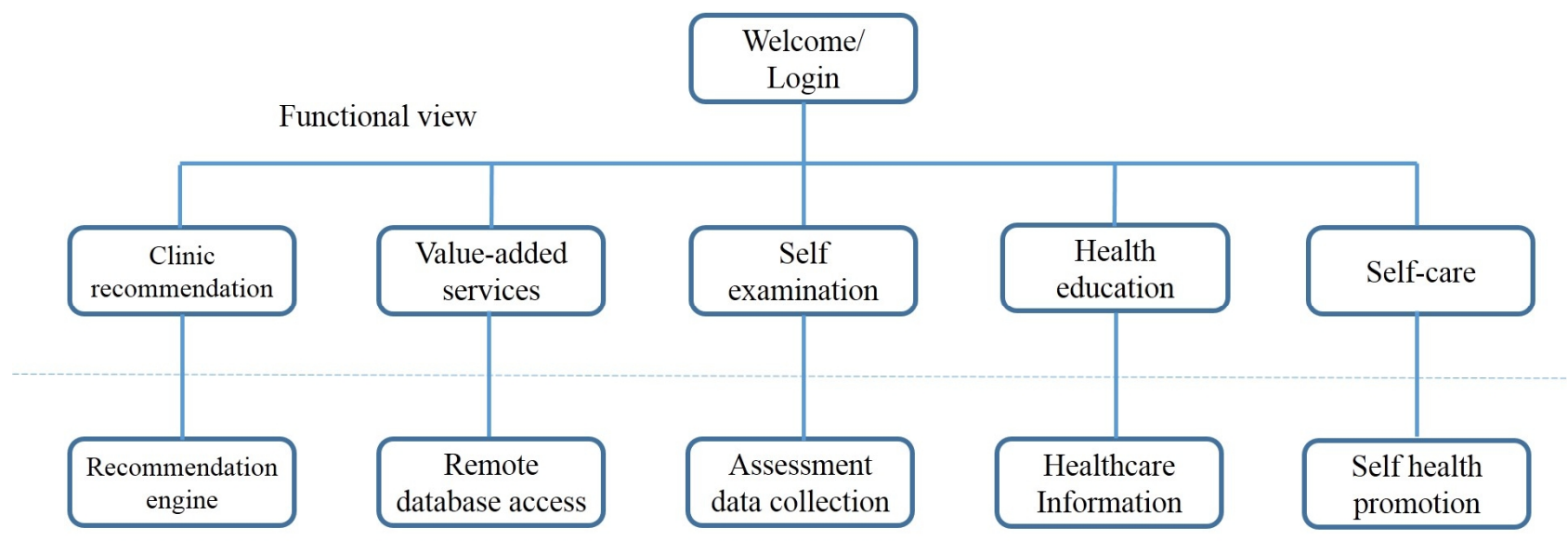

Kernel view

Figure 1. The system structure diagram of App

(a) Clinic registration recommendation system

By observing current on-line self-service registration service provided by the hospital network, newly diagnosed people may be confused when they enter the registration system due to limited awareness of hospital department classification of outpatient disorders then making them unable to distinguish between their disease and the corresponding outpatient clinic. At the same time, no online consultation services for registration in hospital system functions is provided at present time. In this study, the implemented App program will ask the user to click on the body's parts in accordance with the relevant symptoms and location, then display recommended results by fetching the clinics corresponding to database matching result by the disease information. The content of the consultation database contains the types of symptoms of each department and the mapping table of the recommended departments.

(b) Value-added service

At present, in almost all hospitals and examination organizations, people will revisit these places after the medical examination is done, even if all the examination results are normal. It makes many people feel inconvenient. In fact, large-scale medical centers should take the initiative to provide value-added query or inform services about the examination results to the patients to reduce such 'nuisance' medical behavior. Providing this kind of services can bring convenience to patients and reduce the number of seeking medical treatment simultaneously. To implement this, a virtual hospital server is set up to provide the connections form the App, and the above functions are provided through secure remote database access.

(c) Self-examination

Currently there are a variety of Apps on the market to provide health test for self. The collection of these user information and statistical analysis will provide useful reference concepts for both healthcare professionals and the public. There are a variety of self-assessment scales on the Internet, but they are still inconvenient in query because they are individual and distributed. This module attempted to collect all the scales can be searched into a single query interface to facilitate the public to make self-testing at any time.

(d) Health education

For the treatment of disease and self-care, the mobile phone Apps can play an important role on health education. In this study, the health education contents such as wound treatment and rehabilitation knowledge are implemented. It hopes to make users have health conscious at any time, and use the mobile platform to achieve the goal of real-time enrichment.

(e) Self-care 
Once understanding of the individual's health status, how to maintain and exercise for their own become an urgent task. Therefore, the functions of basic life health management for the individual are implemented in this module. It covers the data record, analysis and suggestion of all levels of food and clothing. For example, the self-care of patients with diabetes is very important, the 24-hour daily life health management is indispensable except the calendar to remind medication time and revisiting for examination.

\section{Results}

App implementation. The App system uses Google drive and fusion table for storing the files and data tables. The system allows the authorization-free login mechanism, the user need to create the account and password and enter them in login screen for recording personal health data. All of the functions as implemented can be used after the user accomplishes the login process. The recommendation module first asks the user to enter the sex (male or female) and age (under 14 years of age or adult). These two data values will determine the likelihood weighting of the various diseases. It will then go to different screen depending on whether which part of body the symptoms appears can be clearly pointed out. If the user can point out the affected area, the next screen will ask the user to click the front side of body (including head/eye/ear/nose/throat/mouth/chest/abdomen/hand/foot) or back side of body (including the back and buttocks), then enter the screen which includes all symptoms of selected parts. Once all parts of all the symptoms have been checked, the result will be confirmed by the user and submit to the evaluation module implemented in the App. At present, the adjacent list is adopted to form the cross-reference table for symptoms and clinics lookup. When the user submits the selected symptom data, all the possible clinic departments are stored in a temporary list and it then will be sorted according to the number of symptom. Information about adjacent hospitals corresponding to the suggested clinics will be listed as buttons to provide better convenience to users and enhance the practicality of the App. If the user cannot point out the affected area, the next screen as in Fig. 5 will ask the user to click the symptoms. Once all the symptoms have been checked, the result will be confirmed by the user and submitted to the evaluation module as in previous steps described above.

Usage evaluation. 523 people around the targeted medical center agree to use the App under their admission. The summary about number of hit of each function is listed in Table 1. It shows the clinic recommendation function is the most popular and the required one among all the functions. The number of hit about each department is also recorded in the database, and this number can be as a reference to the department to disseminate general or specific information. On the other hand, the value-added services has the lowest hit number because most people accepted the experiment study knew that it's unable to use in real world which then reduce their will to use this function. It is worth to be mentioned that the functions about self-examination and health education attract people to use with higher frequency which implies people pay attention to their health considerably and the contents of these two functions should be updated frequently to achieve the goal of health promotion and disease prevention.

Table 1. Hit count summary after one month of deployment

\begin{tabular}{|c|c|c|}
\hline & No. of Hit & Percentage \\
\hline Clinic recommendation & 1038 & $30.55 \%$ \\
\hline Value-added services & 106 & $3.12 \%$ \\
\hline Self-examination & 842 & $24.79 \%$ \\
\hline Health education & 796 & $23.43 \%$ \\
\hline Self-care & 615 & $18.12 \%$ \\
\hline
\end{tabular}


To evaluate the effectiveness and satisfaction of the developed system, questionnaire measurement for 376 of 523 people are made after one month usage after they obtained the App. 3 questionnaire are regarded as invalid because of the missing fields, so total amount of 373 questionnaires are collected and the collection rate is $71.3 \%$. In terms of "average" in descriptive statistics, the five-point Likert scales of consent was used in this study. Questionnaire respondents answered three points with no agreement, and four points for agreement. It shows that in Tab. 2 the overall average is 3.78 points and the standard deviation is 0.68 for these 5 functions. For each function in the App system, the highest score is the clinic recommendation with an average of 4.02 points and a standard deviation of less than $1,0.88$. The average of the remaining 4 functions is higher than 3.5 points, and the standard deviation is less than 1, indicating that people have a higher rating and consensus for App health care framework in general. The average and standard deviation of each topic are listed in Table 2.

Table 2. Satisfaction degree summary after one month of deployment

\begin{tabular}{|c|c|c|}
\hline & Mean & $\begin{array}{c}\text { Standard } \\
\text { deviation }\end{array}$ \\
\hline Clinic recommendation & 4.02 & 0.88 \\
\hline Value-added services & 3.51 & $3.12 \%$ \\
\hline Self-examination & 3.86 & $24.79 \%$ \\
\hline Health education & 4.0 & $23.43 \%$ \\
\hline Self-care & 3.58 & $18.12 \%$ \\
\hline
\end{tabular}

\section{Conclusions}

This paper described the implementation of the proposed App program for self-health management, and evaluations are conducted to investigate the acceptance degree of healthcare framework based on mobile devices. The results show that in general people have good evaluation for such a health management model. The future work includes the extension of the functions in the App, such as location-based services, health image management, automatic alarm module and combination of application with Internet of Things.

\section{References}

[1] Information on http://ieknet.iek.org.tw/

[2] Information on http://research2guidance.com/

[3] Information on http://www.iii.org.tw/

[4] Information on http://www.ey.gov.tw/

[5] U.S. Food and Drug Administration: Mobile Medical Applications: Guidance for Industry and Food and Drug Administration Staff (2013) p. 20-22.

[6] E. Dogdu: Semantic web in ehealth, Proceedings of the 47th Annual Southeast Regional Conference, 2009.

[7] Oguz Karan, Canan Bayraktar, Haluk Gumuskaya, and Bekir Karlik: Diagnosing Diabetes Using Neural Networks on Small Mobile Devices, Expert Systems with Applications, Volume 39 (2012) p. 54.

[8] U.S. Food and Drug Administration: Classify Your Medical Device, December 3 (2012). 
[9] Daniel Kramer, Shuai Xu, and Aaron Kesselheim: Regulation of Medical Devices in the United States and European Union, New England Journal of Medicine, Volume 366 (2012) p. 850.

[10] Frederic Resnic and Sharon Lise Normand: Post marketing Surveillance of Medical Devices Filling in the Gaps, New England Journal of Medicine, Volume 366 (2012) p. 875. 